\title{
Asymptotic behavior of an odd-order delay differential equation
}

\author{
Tongxing Li ${ }^{1}$ and Yuriy V Rogovchenko ${ }^{2 *}$ \\ Dedicated to Professor Ivan Kiguradze
}

"Correspondence:

yuriy.rogovchenko@uia.no

${ }^{2}$ Department of Mathematical

Sciences, University of Agder, P.O.

Box 422, Kristiansand, N-4604,

Norway

Full list of author information is

available at the end of the article

\begin{abstract}
We study asymptotic behavior of solutions to a class of odd-order delay differential equations. Our theorems extend and complement a number of related results reported in the literature. An illustrative example is provided.
\end{abstract}

MSC: $34 \mathrm{~K} 11$

Keywords: asymptotic behavior; odd-order; delay differential equation; oscillation

\section{Introduction}

Professor Ivan Kiguradze is widely recognized as one of the leading contemporary experts in the qualitative theory of ordinary differential equations. His research has been partly summarized in the monograph written jointly with Professor Chanturia [1] where many fundamental results on the asymptotic behavior of solutions to important classes of nonlinear differential equations were collected. In particular, the Kiguradze lemma and Kiguradze classes of solutions are well known to researchers working in the area and are extensively used to advance the knowledge further.

In this tribute to Professor Kiguradze, we are concerned with the asymptotic behavior of solutions to an odd-order delay differential equation

$$
\left(r(t)\left(x^{(n-1)}(t)\right)^{\gamma}\right)^{\prime}+p(t)\left(x^{(n-1)}(t)\right)^{\gamma}+q(t) x^{\gamma}(g(t))=0,
$$

where $t \geq t_{0}>0$ and $n \geq 3$ is an odd natural number, $\gamma>0$ is a ratio of odd natural numbers, $r \in C^{1}\left(\left[t_{0}, \infty\right), \mathbb{R}\right), p, q, g \in \mathrm{C}\left(\left[t_{0}, \infty\right), \mathbb{R}\right), r(t)>0, r^{\prime}(t)+p(t) \geq 0, p(t) \geq 0, q(t)>0$, $g(t) \leq t$, and $\lim _{t \rightarrow \infty} g(t)=\infty$.

By a solution of (1.1) we mean a function $x \in \mathrm{C}\left(\left[T_{x}, \infty\right), \mathbb{R}\right), T_{x} \geq t_{0}$, such that $r\left(x^{(n-1)}\right)^{\gamma} \in$ $\mathrm{C}^{1}\left(\left[T_{x}, \infty\right), \mathbb{R}\right)$ and $x(t)$ satisfies $(1.1)$ on $\left[T_{x}, \infty\right)$. We consider only those extendable solutions of (1.1) that do not vanish eventually, that is, condition $\sup \{|x(t)|: t \geq T\}>0$ holds for all $T \geq T_{x}$. We tacitly assume that (1.1) possesses such solutions. As customary, a solution of (1.1) is said to be oscillatory if it has arbitrarily large zeros on the ray $\left[T_{x}, \infty\right)$; otherwise, we call it non-oscillatory.

Analysis of the oscillatory and non-oscillatory behavior of solutions to different classes of differential and functional differential equations has always attracted interest of researchers; see, for instance, [1-19] and the references cited therein. One of the main reasons for this lies in the fact that delay differential equations arise in many applied prob-

\section{Springer}

๑2014 Li and Rogovchenko; licensee Springer. This is an Open Access article distributed under the terms of the Creative Commons Attribution License (http://creativecommons.org/licenses/by/2.0), which permits unrestricted use, distribution, and reproduction in any medium, provided the original work is properly cited. 
lems in natural sciences, technology, and automatic control, $c f$., for instance, Hale [20]. In particular, (1.1) may be viewed as a special case of a more general class of higher-order differential equations with a one-dimensional $p$-Laplacian, which, as mentioned by Agarwal et al. [4], have applications in continuum mechanics.

Let us briefly comment on a number of closely related results which motivated our study. In $[2,5-8,14]$, the authors investigated asymptotic properties of a third-order delay differential equation

$$
\left(r(t) x^{\prime \prime}(t)\right)^{\prime}+p(t) x^{\prime}(t)+q(t) x(\sigma(t))=0 .
$$

Using a Riccati substitution, Liu et al. [11], Zhang et al. [16], and Zhang et al. [18] studied oscillation of (1.1) assuming that $n \geq 2$ is even, $g(t) \leq t$, and

$$
\int_{T_{1}}^{\infty}\left[\frac{1}{r(s)} \exp \left(-\int_{T}^{s} \frac{p(\tau)}{r(\tau)} \mathrm{d} \tau\right)\right]^{1 / \gamma} \mathrm{d} s=\infty, \quad \text { for } T_{1} \geq T \geq t_{0}
$$

In the special case when $p(t)=0,(1.1)$ reduces to a two-term differential equation

$$
\left(r(t)\left(x^{(n-1)}(t)\right)^{\gamma}\right)^{\prime}+q(t) x^{\gamma}(g(t))=0
$$

which was studied by Zhang et al. [17] who established the following result.

Theorem 1.1 ([17, Corollary 2.1]) Let

$$
\delta(t):=\int_{t}^{\infty} r^{-1 / \gamma}(s) \mathrm{d} s
$$

and assume that $\delta\left(t_{0}\right)<\infty$. Suppose also that

$$
\frac{1}{((n-1) !)^{\gamma}} \liminf _{t \rightarrow \infty} \int_{g(t)}^{t} q(s)\left(\frac{g^{n-1}(s)}{r^{1 / \gamma}(g(s))}\right)^{\gamma} \mathrm{d} s>\frac{1}{\mathrm{e}}
$$

and, for some $\lambda_{1} \in(0,1)$,

$$
\limsup _{t \rightarrow \infty} \int_{t_{0}}^{t}\left[q(s)\left(\frac{\lambda_{1} g^{n-2}(s)}{(n-2) !}\right)^{\gamma} \delta^{\gamma}(s)-\frac{\gamma^{\gamma+1}}{(\gamma+1)^{\gamma+1} \delta(s) r^{1 / \gamma}(s)}\right] \mathrm{d} s=\infty .
$$

Then every solution of (1.3) is either oscillatory or converges to zero as $t \rightarrow \infty$.

To the best of our knowledge, only a few results are known regarding oscillation of (1.1) for $n$ odd. Furthermore, in this case the methods in $[11,18]$ which employ Riccati substitutions cannot be applied to the analysis of (1.1). Therefore, the objective of this paper is to extend the techniques exploited in [17] to the study of (1.1) in the case when the integral in (1.2) is finite, that is, for all $T_{1} \geq T \geq t_{0}$,

$$
\int_{T_{1}}^{\infty}\left[\frac{1}{r(s)} \exp \left(-\int_{T}^{s} \frac{p(\tau)}{r(\tau)} \mathrm{d} \tau\right)\right]^{1 / \gamma} \mathrm{d} s<\infty .
$$

As usual, all functional inequalities considered in this paper are supposed to hold for all $t$ large enough. Without loss of generality, we may deal only with positive solutions of (1.1), 
because under our assumption that $\gamma$ is a ratio of odd natural numbers, if $x(t)$ is a solution of (1.1), so is $-x(t)$.

\section{Main results}

We need the following auxiliary lemmas.

Lemma 2.1 Assume that (1.2) is satisfied and let $x(t)$ be an eventually positive solution of (1.1). Then there exists a sufficiently large $t_{1} \geq t_{0}$ such that, for all $t \geq t_{1}$,

$$
x(t)>0, \quad x^{(n-1)}(t)>0, \quad x^{(n)}(t)<0 .
$$

Proof Let $x(t)$ be an eventually positive solution of (1.1). Then there exists a $T_{0} \geq t_{0}$ such that $x(t)>0$ and $x(g(t))>0$ for all $t \geq T_{0}$. By virtue of (1.1),

$$
\left(r(t)\left(x^{(n-1)}(t)\right)^{\gamma}\right)^{\prime}+p(t)\left(x^{(n-1)}(t)\right)^{\gamma}<0 .
$$

Thus,

$$
\left(\exp \left(\int_{t_{0}}^{t} \frac{p(\tau)}{r(\tau)} \mathrm{d} \tau\right) r(t)\left(x^{(n-1)}(t)\right)^{\gamma}\right)^{\prime}<0,
$$

which means that the function

$$
\exp \left(\int_{t_{0}}^{t} \frac{p(\tau)}{r(\tau)} \mathrm{d} \tau\right) r(t)\left(x^{(n-1)}(t)\right)^{\gamma}
$$

is decreasing for $t \geq T_{0}$. Therefore, $x^{(n-1)}(t)$ does not change sign eventually, that is, there exists a $t_{1} \geq T_{0}$ such that either $x^{(n-1)}(t)>0$ or $x^{(n-1)}(t)<0$ for all $t \geq t_{1}$.

We claim that $x^{(n-1)}(t)>0$ for all $t \geq t_{1}$. Otherwise, there should exist a $T \geq t_{1}$ such that

$$
\exp \left(\int_{t_{0}}^{T} \frac{p(\tau)}{r(\tau)} \mathrm{d} \tau\right) r(T)\left(x^{(n-1)}(T)\right)^{\gamma}=M \exp \left(\int_{t_{0}}^{T} \frac{p(\tau)}{r(\tau)} \mathrm{d} \tau\right)<0
$$

and, for all $t \geq T$,

$$
\exp \left(\int_{t_{0}}^{t} \frac{p(\tau)}{r(\tau)} \mathrm{d} \tau\right) r(t)\left(x^{(n-1)}(t)\right)^{\gamma} \leq M \exp \left(\int_{t_{0}}^{T} \frac{p(\tau)}{r(\tau)} \mathrm{d} \tau\right)<0,
$$

where

$$
M:=r(T)\left(x^{(n-1)}(T)\right)^{\gamma} .
$$

Inequality (2.3) yields

$$
x^{(n-1)}(t) \leq M^{1 / \gamma}\left[\frac{1}{r(t)} \exp \left(-\int_{T}^{t} \frac{p(\tau)}{r(\tau)} \mathrm{d} \tau\right)\right]^{1 / \gamma} .
$$

Integrating this inequality from $T_{1}$ to $t, T_{1} \geq T$, we conclude that

$$
x^{(n-2)}(t) \leq x^{(n-2)}\left(T_{1}\right)+M^{1 / \gamma} \int_{T_{1}}^{t}\left[\frac{1}{r(s)} \exp \left(-\int_{T}^{s} \frac{p(\tau)}{r(\tau)} \mathrm{d} \tau\right)\right]^{1 / \gamma} \mathrm{d} s .
$$


Passing to the limit as $t \rightarrow \infty$ and using (1.2), we deduce that

$$
\lim _{t \rightarrow \infty} x^{(n-2)}(t)=-\infty
$$

It follows now from the inequalities $x^{(n-1)}(t)<0$ and $x^{(n-2)}(t)<0$ that $x(t)<0$, which contradicts our assumption that $x(t)>0$. Finally, write (2.2) in the form

$$
\begin{aligned}
& \exp \left(\int_{t_{0}}^{t} \frac{p(\tau)}{r(\tau)} \mathrm{d} \tau\right)\left[r^{\prime}(t)+p(t)\right]\left(x^{(n-1)}(t)\right)^{\gamma} \\
& \quad+\gamma r(t) \exp \left(\int_{t_{0}}^{t} \frac{p(\tau)}{r(\tau)} \mathrm{d} \tau\right)\left(x^{(n-1)}(t)\right)^{\gamma-1} x^{(n)}(t)<0,
\end{aligned}
$$

which implies that $x^{(n)}(t)<0$. This completes the proof.

Lemma 2.2 (Agarwal et al. [3]) Assume that $u \in \mathrm{C}^{n}\left(\left[t_{0}, \infty\right), \mathbb{R}^{+}\right), u^{(n)}(t)$ is non-positive for all large $t$ and not identically zero on $\left[t_{0}, \infty\right)$. If $\lim _{t \rightarrow \infty} u(t) \neq 0$, then for every $\lambda \in(0,1)$, there exists a $t_{\lambda} \in\left[t_{0}, \infty\right)$ such that

$$
u(t) \geq \frac{\lambda}{(n-1) !} t^{n-1}\left|u^{(n-1)}(t)\right|
$$

holds on $\left[t_{\lambda}, \infty\right)$.

Lemma 2.3 (Agarwal et al. [4]) The equation

$$
\left(r(t)\left(x^{\prime}(t)\right)^{\gamma}\right)^{\prime}+a(t) x^{\gamma}(t)=0,
$$

where $\gamma>0$ is a quotient of odd natural numbers, $r \in C^{1}\left(\left[t_{0}, \infty\right),(0, \infty)\right)$, and $a \in$ $\mathrm{C}\left(\left[t_{0}, \infty\right), \mathbb{R}\right)$ is non-oscillatory if and only if there exist a number $T \geq t_{0}$ and a function $v \in C^{1}([T, \infty), \mathbb{R})$ such that, for all $t \geq T$,

$$
v^{\prime}(t)+\gamma \frac{v^{(\gamma+1) / \gamma}(t)}{r^{1 / \gamma}(t)}+a(t) \leq 0
$$

For a compact presentation of our results, we introduce the following notation:

$$
\begin{aligned}
& E(k, l):=\exp \left(\int_{k}^{l} \frac{p(\tau)}{r(\tau)} \mathrm{d} \tau\right), \quad \delta(t):=\int_{t}^{\infty} \frac{\mathrm{d} s}{\left(r(s) E\left(t_{0}, s\right)\right)^{1 / \gamma}}, \\
& \varphi(t):=\frac{p(t)}{r(t)}+\frac{\gamma^{\gamma+1}}{(\gamma+1)^{\gamma+1}} \frac{\phi_{+}^{\gamma+1}(t) E\left(t_{0}, t\right)}{\delta(t) r^{1 / \gamma}(t)}, \\
& \phi(t):=\frac{1}{E^{1 / \gamma}\left(t_{0}, t\right)}-\frac{1}{\gamma} \delta(t) p(t) r^{(1-\gamma) / \gamma}(t), \quad \phi_{+}(t):=\max [0, \phi(t)] .
\end{aligned}
$$

Theorem 2.4 Assume that

$$
\frac{1}{((n-1) !)^{\gamma}} \liminf _{t \rightarrow \infty} \int_{g(t)}^{t} \frac{q(s)}{r(g(s))}\left(g^{n-1}(s)\right)^{\gamma} E(g(s), s) \mathrm{d} s>\frac{1}{\mathrm{e}} .
$$


Then every solution $x(t)$ of $(1.1)$ is either oscillatory or satisfies

$$
\lim _{t \rightarrow \infty} x(t)=0
$$

provided that either

(i) (1.2) holds or

(ii) (1.4) is satisfied and, for some $\lambda_{1} \in(0,1)$,

$$
\limsup _{t \rightarrow \infty} \int_{t_{0}}^{t}\left[q(s)\left(\frac{\lambda_{1}}{(n-2) !} g^{n-2}(s) \delta(s)\right)^{\gamma} E\left(t_{0}, s\right)-\varphi(s)\right] \mathrm{d} s=\infty .
$$

Proof Assume that (1.1) has a non-oscillatory solution $x(t)$ which is eventually positive and such that

$$
\lim _{t \rightarrow \infty} x(t) \neq 0 .
$$

Case (i) By Lemma 2.1, we conclude that (2.1) holds for all $t \geq t_{1}$, where $t_{1} \geq t_{0}$ is sufficiently large. It follows from Lemma 2.2 that

$$
x(t) \geq \frac{\lambda t^{n-1}}{(n-1) !} x^{(n-1)}(t)=\frac{\lambda t^{n-1}}{(n-1) ! r^{1 / \gamma}(t)} r^{1 / \gamma}(t) x^{(n-1)}(t),
$$

for every $\lambda \in(0,1)$ and for all sufficiently large $t$. Let

$$
y(t):=r(t)\left(x^{(n-1)}(t)\right)^{\gamma} .
$$

By virtue of (1.1), we conclude that $y(t)$ is a positive solution of a differential inequality

$$
y^{\prime}(t)+\frac{p(t)}{r(t)} y(t)+q(t)\left(\frac{\lambda g^{n-1}(t)}{(n-1) ! r^{1 / \gamma}(g(t))}\right)^{\gamma} y(g(t)) \leq 0 .
$$

However, it follows from the result due to Werbowski [15, Corollary 1] that the latter inequality does not have positive solutions under the assumption (2.4), which is a contradiction. The proof of part (i) is complete.

Case (ii) Similar analysis to that in Lemma 2.1 leads to the conclusion that a nonoscillatory positive solution with the property (2.7) satisfies, for $t \geq t_{1}$, either conditions (2.1) or

$$
x(t)>0, \quad x^{(n-2)}(t)>0, \quad x^{(n-1)}(t)<0,
$$

where $t_{1} \geq t_{0}$ is sufficiently large. Assume first that (2.1) holds. As in the proof of the part (i), one arrives at a contradiction with the condition (2.4). Suppose now that (2.8) holds. For $t \geq t_{1}$, define a new function $v(t)$ by

$$
v(t):=\frac{r(t)\left(x^{(n-1)}(t)\right)^{\gamma}}{\left(x^{(n-2)}(t)\right)^{\gamma}} .
$$

Then $v(t)<0$ for $t \geq t_{1}$. Since

$$
\left(r(t)\left(x^{(n-1)}(t)\right)^{\gamma} E\left(t_{0}, t\right)\right)^{\prime}=-q(t) x^{\gamma}(g(t)) E\left(t_{0}, t\right)<0,
$$


we deduce that the function $r(t)\left(x^{(n-1)}(t)\right)^{\gamma} E\left(t, t_{0}\right)$ is decreasing. Thus, for $s \geq t \geq t_{1}$,

$$
\left(r(s) E\left(t_{0}, s\right)\right)^{1 / \gamma} x^{(n-1)}(s) \leq\left(r(t) E\left(t_{0}, t\right)\right)^{1 / \gamma} x^{(n-1)}(t) .
$$

Dividing both sides of $(2.10)$ by $\left(r(s) E\left(t_{0}, s\right)\right)^{1 / \gamma}$ and integrating the resulting inequality from $t$ to $T$, we obtain

$$
x^{(n-2)}(T) \leq x^{(n-2)}(t)+\left(r(t) E\left(t_{0}, t\right)\right)^{1 / \gamma} x^{(n-1)}(t) \int_{t}^{T} \frac{\mathrm{d} s}{\left(r(s) E\left(t_{0}, s\right)\right)^{1 / \gamma}} .
$$

Letting $T \rightarrow \infty$ and taking into account that $x^{(n-1)}(t)<0$ and $x^{(n-2)}(t)>0$, we conclude that

$$
\lim _{T \rightarrow \infty} x^{(n-2)}(T) \geq 0
$$

Hence,

$$
0 \leq x^{(n-2)}(t)+\left(r(t) E\left(t_{0}, t\right)\right)^{1 / \gamma} x^{(n-1)}(t) \delta(t)
$$

which yields

$$
-\frac{x^{(n-1)}(t)}{x^{(n-2)}(t)} \delta(t)\left(r(t) E\left(t_{0}, t\right)\right)^{1 / \gamma} \leq 1 .
$$

Thus, by (2.9), we conclude that

$$
-v(t) \delta^{\gamma}(t) E\left(t_{0}, t\right) \leq 1
$$

Differentiation of (2.9) yields

$$
v^{\prime}(t)=\frac{\left(r(t)\left(x^{(n-1)}(t)\right)^{\gamma}\right)^{\prime}}{\left(x^{(n-2)}(t)\right)^{\gamma}}-\gamma \frac{r(t)\left(x^{(n-1)}(t)\right)^{\gamma+1}}{\left(x^{(n-2)}(t)\right)^{\gamma+1}} .
$$

It follows now from (1.1) and (2.9) that

$$
v^{\prime}(t)=-p(t) \frac{v(t)}{r(t)}-q(t) \frac{x^{\gamma}(g(t))}{\left(x^{(n-2)}(t)\right)^{\gamma}}-\gamma \frac{v^{(\gamma+1) / \gamma}(t)}{r^{1 / \gamma}(t)} .
$$

On the other hand, it follows from Lemma 2.2 that

$$
x(t) \geq \frac{\lambda}{(n-2) !} t^{n-2} x^{(n-2)}(t),
$$

for every $\lambda \in(0,1)$ and for all sufficiently large $t$. Therefore, (2.11) yields

$$
\begin{aligned}
\nu^{\prime}(t) & \leq \frac{p(t)}{r(t) \delta^{\gamma}(t) E\left(t_{0}, t\right)}-q(t)\left(\frac{x(g(t))}{x^{(n-2)}(g(t))}\right)^{\gamma}\left(\frac{x^{(n-2)}(g(t))}{x^{(n-2)}(t)}\right)^{\gamma}-\gamma\left(\frac{v^{\gamma+1}(t)}{r(t)}\right)^{1 / \gamma} \\
& \leq \frac{p(t)}{r(t) \delta^{\gamma}(t) E\left(t_{0}, t\right)}-q(t)\left(\frac{\lambda}{(n-2) !} g^{n-2}(t)\right)^{\gamma}-\gamma\left(\frac{v^{\gamma+1}(t)}{r(t)}\right)^{1 / \gamma} .
\end{aligned}
$$


Multiplying (2.12) by $\delta^{\gamma}(t) E\left(t_{0}, t\right)$ and integrating the resulting inequality from $t_{1}$ to $t$, we have

$$
\begin{gathered}
\delta^{\gamma}(t) E\left(t_{0}, t\right) v(t)-\delta^{\gamma}\left(t_{1}\right) E\left(t_{0}, t_{1}\right) v\left(t_{1}\right)-\int_{t_{1}}^{t} \frac{p(s)}{r(s)} \mathrm{d} s \\
+\gamma \int_{t_{1}}^{t} r^{-1 / \gamma}(s) \delta^{\gamma-1}(s) E\left(t_{0}, s\right) \phi_{+}(s) v(s) \mathrm{d} s \\
+\int_{t_{1}}^{t} q(s)\left(\frac{\lambda}{(n-2) !} g^{n-2}(s)\right)^{\gamma} \delta^{\gamma}(s) E\left(t_{0}, s\right) \mathrm{d} s \\
+\int_{t_{1}}^{t} \gamma\left(\frac{\nu^{\gamma+1}(s)}{r(s)}\right)^{1 / \gamma} \delta^{\gamma}(s) E\left(t_{0}, s\right) \mathrm{d} s \leq 0 .
\end{gathered}
$$

Let $A:=\delta^{\gamma}(s) E\left(t_{0}, s\right) r^{-1 / \gamma}(s)$ and $B:=r^{-1 / \gamma}(s) \delta^{\gamma-1}(s) E\left(t_{0}, s\right) \phi_{+}(s)$. Using the fact that $v^{(\gamma+1) / \gamma}(s)=(-\gamma(s))^{(\gamma+1) / \gamma}$ and the inequality

$$
-B v(s)-A v^{(\gamma+1) / \gamma}(s) \leq \frac{\gamma^{\gamma}}{(\gamma+1)^{\gamma+1}} \frac{B^{\gamma+1}}{A^{\gamma}}, \quad A>0
$$

(see Zhang and Wang [19, Lemma 2.3] for details) and the definition of $\varphi$, we derive from (2.11) that

$$
\int_{t_{1}}^{t}\left[q(s)\left(\frac{\lambda}{(n-2) !} g^{n-2}(s)\right)^{\gamma} \delta^{\gamma}(s) E\left(t_{0}, s\right)-\varphi(s)\right] \mathrm{d} s \leq \delta^{\gamma}\left(t_{1}\right) E\left(t_{0}, t_{1}\right) v\left(t_{1}\right)+1,
$$

which contradicts (2.6). This completes the proof for the part (ii).

Remark 2.5 For a result similar to the one established in part (i) in Theorem 2.4, see also Zhang et al. [16, Theorem 5.3].

Remark 2.6 For $p(t)=0$, Theorem 2.4 includes Theorem 1.1.

In the remainder of this section, we use different approaches to arrive at the conclusion of Theorem 2.4. First, we employ the integral averaging technique to replace assumption (2.6) with a Philos-type condition.

To this end, let $\mathbb{D}=\left\{(t, s): t \geq s \geq t_{0}\right\}$. We say that a function $H \in C(\mathbb{D}, \mathbb{R})$ belongs to the class $\mathcal{P}_{\gamma}$ if

$$
H(t, t)=0, \quad \text { for } t \geq t_{0}, \quad H(t, s)>0, \quad \text { for } t>s \geq t_{0},
$$

and $H$ has a non-positive continuous partial derivative $\partial H / \partial s$ with respect to the second variable satisfying the condition

$$
-\frac{\partial}{\partial s} H(t, s)=\xi(t, s) H^{\gamma /(\gamma+1)}(t, s)
$$

for some function $\xi \in L_{\mathrm{loc}}(\mathbb{D}, \mathbb{R})$. 
Theorem 2.7 Let $\delta(t)$ be as in Theorem 2.4 and suppose that (1.4) and (2.4) hold. Assume that there exists a function $H \in \mathcal{P}_{\gamma}$ such that

$$
\begin{aligned}
\limsup _{t \rightarrow \infty} \int_{t_{1}}^{t}\left[H(t, s) q(s)\left(\frac{\lambda_{1}}{(n-2) !} g^{n-2}(s)\right)^{\gamma}\right. \\
\left.-\frac{H(t, s) p(s)}{r(s) \delta^{\gamma}(s) E\left(t_{0}, s\right)}-\frac{r(s)(\xi(t, s))^{\gamma+1}}{(\gamma+1)^{\gamma+1}}\right] \mathrm{d} s>0,
\end{aligned}
$$

for all $t_{1} \geq t_{0}$ and for some $\lambda_{1} \in(0,1)$. Then the conclusion of Theorem 2.4 remains intact.

Proof Assuming that $x(t)$ is an eventually positive solution of (1.1) that satisfies (2.7) and proceeding as in the proof of Theorem 2.4, we arrive at the inequality (2.12) which holds for all $\lambda \in(0,1)$. Multiplying $(2.12)$ by $H(t, s)$ and integrating the resulting inequality from $t_{1}$ to $t$, we obtain

$$
\begin{aligned}
& \int_{t_{1}}^{t} H(t, s)\left[q(s)\left(\frac{\lambda g^{n-2}(s)}{(n-2) !}\right)^{\gamma}-\frac{p(s)}{r(s) \delta \gamma(s) E\left(t_{0}, s\right)}\right] \mathrm{d} s \\
& \quad \leq H\left(t, t_{1}\right) v\left(t_{1}\right)+\int_{t_{1}}^{t} \frac{\partial H(t, s)}{\partial s} v(s) \mathrm{d} s-\int_{t_{1}}^{t} \gamma H(t, s) \frac{v^{(\gamma+1) / \gamma}(s)}{r^{1 / \gamma}(s)} \mathrm{d} s \\
& \quad=H\left(t, t_{1}\right) v\left(t_{1}\right)-\int_{t_{1}}^{t} \xi(t, s) H^{\gamma /(\gamma+1)}(t, s) v(s) \mathrm{d} s-\int_{t_{1}}^{t} \gamma H(t, s) \frac{v^{(\gamma+1) / \gamma}(s)}{r^{1 / \gamma}(s)} \mathrm{d} s .
\end{aligned}
$$

Let

$$
A:=\left(\gamma H(t, s) \frac{(-v(s))^{(\gamma+1) / \gamma}}{r^{1 / \gamma}(s)}\right)^{\gamma /(\gamma+1)}
$$

and

$$
B:=\left(\frac{\gamma \xi(t, s) r^{1 /(\gamma+1)}(s)}{(\gamma+1) \gamma^{\gamma /(\gamma+1)}}\right)^{\gamma}
$$

Using the inequality

$$
\frac{\gamma+1}{\gamma} A B^{1 / \gamma}-A^{(\gamma+1) / \gamma} \leq \frac{1}{\gamma} B^{(\gamma+1) / \gamma},
$$

we obtain

$$
\begin{aligned}
& \int_{t_{1}}^{t}\left[H(t, s) q(s)\left(\frac{\lambda}{(n-2) !} g^{n-2}(s)\right)^{\gamma}-\frac{H(t, s) p(s)}{r(s) \delta^{\gamma}(s) E\left(t_{0}, s\right)}-\frac{r(s) \xi^{\gamma+1}(t, s)}{(\gamma+1)^{\gamma+1}}\right] \mathrm{d} s \\
& \quad \leq H\left(t, t_{1}\right) v\left(t_{1}\right)<0,
\end{aligned}
$$

which contradicts assumption (2.13). This completes the proof.

Finally, we formulate also a comparison result for (1.1) that leads to the conclusion of Theorem 2.4. 
Theorem 2.8 Let $\delta(t)$ be as above, and assume that (1.4) and (2.4) hold. If a second-order half-linear ordinary differential equation

$$
\left(r(t)\left(u^{\prime}(t)\right)^{\gamma}\right)^{\prime}+\left[q(t)\left(\frac{\lambda_{1}}{(n-2) !} g^{n-2}(t)\right)^{\gamma}-\frac{p(t)}{r(t) \delta^{\gamma}(t) E\left(t_{0}, t\right)}\right] u^{\gamma}(t)=0
$$

is oscillatory for some $\lambda_{1} \in(0,1)$, then the conclusion of Theorem 2.4 remains intact.

Proof Assuming again that $x(t)$ is an eventually positive solution of (1.1) that satisfies (2.7) and proceeding as in the proof of Theorem 2.4, we obtain (2.12) which holds for all $\lambda \in(0,1)$. By virtue of Lemma 2.3 , we conclude that $(2.14)$ is non-oscillatory, which is a contradiction. The proof is complete.

\section{Example}

The following example illustrates possible applications of theoretical results obtained in the previous section.

Example 3.1 For $t \geq 1$, consider the third-order differential equation

$$
\left(t x^{\prime \prime}(t)\right)^{\prime}+x^{\prime \prime}(t)+\frac{t-2}{\mathrm{e}^{2}} x(t-2)=0
$$

It is not difficult to verify that (1.4) holds and

$$
\begin{aligned}
& \liminf _{t \rightarrow \infty} \int_{g(t)}^{t} q(s)\left(\frac{g^{n-1}(s)}{r^{1 / \gamma}(g(s))}\right)^{\gamma} \exp \left(\int_{g(s)}^{s} \frac{p(v)}{r(v)} \mathrm{d} v\right) \mathrm{d} s \\
& =\frac{1}{\mathrm{e}^{2}} \liminf _{t \rightarrow \infty} \int_{t-2}^{t} s(s-2) \mathrm{d} s=\infty .
\end{aligned}
$$

Let $t_{0}=1$. Then $\delta(t)=1 / t, \phi(t)=0, \varphi(t)=1 / t$, and thus

$$
\begin{aligned}
& \limsup _{t \rightarrow \infty} \int_{t_{0}}^{t}\left[q(s)\left(\frac{\lambda_{1} g^{n-2}(s) \delta(s)}{(n-2) !}\right)^{\gamma} \exp \left(\int_{t_{0}}^{s} \frac{p(\tau)}{r(\tau)} \mathrm{d} \tau\right)-\varphi(s)\right] \mathrm{d} s \\
& \quad=\limsup _{t \rightarrow \infty} \int_{1}^{t}\left[\lambda_{1} \frac{(s-2)^{2}}{\mathrm{e}^{2}}-\frac{1}{s}\right] \mathrm{d} s=\infty,
\end{aligned}
$$

for some $\lambda_{1} \in(0,1)$. Hence, by Theorem 2.4, every solution of (3.1) is either oscillatory or satisfies (2.5). As a matter of fact, $x(t)=\mathrm{e}^{-t}$ is a solution of this equation satisfying condition (2.5).

Remark 3.2 Note that Theorems 2.4, 2.7, and 2.8 ensure that every solution $x(t)$ of (1.1) is either oscillatory or satisfies (2.5) and, unfortunately, these results cannot distinguish solutions with different behaviors. Since the sign of the derivative $x^{\prime}(t)$ is not known, it is difficult to establish sufficient conditions which guarantee that all solutions of (1.1) are just oscillatory and do not satisfy (2.5). Neither is it possible to use the technique exploited in this paper for proving that all solutions of (1.1) satisfy (2.5). Therefore, these two interesting problems remain for future research. 


\section{Competing interests}

The authors declare that they have no competing interests.

\section{Authors' contributions}

Both authors contributed equally to this work and are listed in alphabetical order. They both read and approved the final version of the manuscript.

\section{Author details}

${ }^{1}$ Qingdao Technological University, Feixian, Shandong 273400, P.R. China. ${ }^{2}$ Department of Mathematical Sciences, University of Agder, P.O. Box 422, Kristiansand, N-4604, Norway.

\section{Acknowledgements}

The authors express their sincere gratitude to both anonymous referees for the careful reading of the original manuscript and useful comments that helped to improve the presentation of the results and accentuate important details.

Received: 29 January 2014 Accepted: 22 April 2014 Published: 09 May 2014

\section{References}

1. Kiguradze, IT, Chanturia, TA: Asymptotic Properties of Solutions of Nonautonomous Ordinary Differential Equations. Kluwer Academic, Dordrecht (1993). Translated from the 1985 Russian original

2. Agarwal, RP, Aktas, MF, Tiryaki, A: On oscillation criteria for third order nonlinear delay differential equations. Arch. Math. 45, 1-18 (2009)

3. Agarwal, RP, Grace, SR, O'Regan, D: Oscillation Theory for Difference and Functional Differential Equations. Kluwer Academic, Dordrecht (2000)

4. Agarwal, RP, Grace, SR, O'Regan, D: Oscillation Theory for Second Order Linear, Half-Linear, Superlinear and Sublinear Dynamic Equations. Kluwer Academic, Dordrecht (2002)

5. Baculíková, B, Džurina, J: Comparison theorems for the third-order delay trinomial differential equations. Adv. Differ. Equ. 2010, 160761 (2010)

6. Baculíková, B, Džurina, J, Rogovchenko, YuV: Oscillation of third order trinomial delay differential equations. Appl. Math. Comput. 218, 7023-7033 (2012)

7. Džurina, J, Komariková, R: Asymptotic properties of third-order delay trinomial differential equations. Abstr. Appl. Anal. 2011, 730128 (2011)

8. Džurina, J, Kotorová, R: Properties of the third order trinomial differential equations with delay argument. Nonlinear Anal. TMA 71, 1995-2002 (2009)

9. Grace, SR, Agarwal, RP, Pavani, R, Thandapani, E: On the oscillation of certain third order nonlinear functional differential equations. Appl. Math. Comput. 202, 102-112 (2008)

10. Li, T, Rogovchenko, YuV, Tang, S: Oscillation of second-order nonlinear differential equations with damping. Math. Slovaca (2014, in press)

11. Liu, S, Zhang, Q, Yu, Y: Oscillation of even-order half-linear functional differential equations with damping. Comput. Math. Appl. 61, 2191-2196 (2011)

12. Philos, ChG: Oscillation theorems for linear differential equations of second order. Arch. Math. 53, 482-492 (1989)

13. Rogovchenko, YuV, Tuncay, F: Oscillation criteria for second-order nonlinear differential equations with damping. Nonlinear Anal. 69, 208-221 (2008)

14. Tiryaki, A, Aktas, MF: Oscillation criteria of a certain class of third order nonlinear delay differential equations with damping. J. Math. Anal. Appl. 325, 54-68 (2007)

15. Werbowski, J: Oscillations of first-order differential inequalities with deviating arguments. Ann. Mat. Pura Appl. 140 383-392 (1985)

16. Zhang, C, Agarwal, RP, Li, T: Oscillation and asymptotic behavior of higher-order delay differential equations with p-Laplacian like operators. J. Math. Anal. Appl. 409, 1093-1106 (2014)

17. Zhang, C, Li, T, Sun, B, Thandapani, E: On the oscillation of higher-order half-linear delay differential equations. Appl. Math. Lett. 24, 1618-1621 (2011)

18. Zhang, Q, Liu, S, Gao, L: Oscillation criteria for even-order half-linear functional differential equations with damping Appl. Math. Lett. 24, 1709-1715 (2011)

19. Zhang, S, Wang, Q: Oscillation of second-order nonlinear neutral dynamic equations on time scales. Appl. Math. Comput. 216, 2837-2848 (2010)

20. Hale, JK: Theory of Functional Differential Equations. Springer, New York (1977)

10.1186/1687-2770-2014-107

Cite this article as: Li and Rogovchenko: Asymptotic behavior of an odd-order delay differential equation. Boundary Value Problems 2014, 2014:107 